\title{
Effect of Agronomic Biofortification with Zinc and Iron on Yield and Quality of Pearlmillet [Pennisetum glaucum (L.)] Genotypes
}

\author{
Sharanappa", H. S. Latha, B. K. Desai, B. G.Koppalkar and M. V. Ravi \\ Department of Agronomy, College of Agriculture, UAS, Raichur, \\ Karnataka, India-584104, India \\ *Corresponding author
}

\section{Keywords \\ Pearlmillet, stover and grain yield, zinc and iron content \\ Article Info \\ Accepted: \\ 15 August 2019 \\ Available Online: \\ 10 September 2019}

\section{A B S T R A C T}

A field experiment was conducted at Agricultural College farm, Raichur, Karnataka, during kharif 2016-2017 to study the effect of agronomic biofortification with zinc and iron on yield and quality of pearlmillet [Pennisetum glaucum (L.)] genotypes, to evaluate and analysis of pearlmillet genotypes through agronomic biofortification to achieve higher grain yield and quality parameters. The genotype $\mathrm{G}_{3}$ : $\mathrm{HFeZn}-113$ (high in $\mathrm{Zn} \mathrm{\&} \mathrm{Fe}$ ) recorded significantly higher grain and stover yield $\left(1721 \mathrm{~kg} \mathrm{ha}^{-1}\right.$ and $4437 \mathrm{~kg} \mathrm{ha}^{-1}$, respectively) and among the micronutrient application significantly higher grain and stover yield of pearlmillet was obtained in $\mathrm{M}_{7}$ : soil application of $\mathrm{ZnSO}_{4} @ 15 \mathrm{~kg} \mathrm{ha}^{-1}$ \& $\mathrm{FeSO}_{4}$ @ $10 \mathrm{~kg} \mathrm{ha}^{-1}+$ Foliar application of $0.5 \% \mathrm{ZnSO}_{4}$ and $\mathrm{FeSO}_{4}\left(1904 \mathrm{~kg} \mathrm{ha}^{-1}\right.$ and $4611 \mathrm{~kg}$ $\mathrm{ha}^{-1}$, respectively). The genotype $\mathrm{G}_{3}$ : $\mathrm{HFeZn}-113$ (high in $\mathrm{Zn} \& \mathrm{Fe}$ ) significantly higher number of ear heads, length of ear heads, weight of ear head and grain weight in $\mathrm{G}_{3}$ : HFeZn-113 (high in Zn \& Fe) $\left(3.21,14.84 \mathrm{~cm}, 39.37\right.$ and $33.84 \mathrm{~g} \mathrm{plant}^{-1}$, respectively). Among the micronutrient application higher number of ear heads, length of ear heads, weight of ear head and grain weight recorded with $\mathrm{M}_{7}$ : Soil application of $\mathrm{ZnSO}_{4} @ 15 \mathrm{~kg}$ $\mathrm{ha}^{-1} \& \mathrm{FeSO}_{4} @ 10 \mathrm{~kg} \mathrm{ha}^{-1}+$ Foliar application of $0.5 \% \mathrm{ZnSO}_{4}$ and $\mathrm{FeSO}_{4}$ each $(5.45$, $20.41 \mathrm{~cm} 48.09$ and $39.89 \mathrm{~g}$ plant $^{-1}$, respectively). The genotype $\mathrm{G}_{3}: \mathrm{HFeZn}-113$ (high in $\mathrm{Zn} \& \mathrm{Fe}$ ) recorded significantly higher zinc content $(29.56,38.89$ and $66.45 \mathrm{ppm}$ in grain, stover and total zinc content, respectively). Among micronutrients application, significantly higher zinc content was observed in $\mathrm{M}_{7}$ : Soil application of $\mathrm{ZnSO}_{4} @ 15 \mathrm{~kg}$ $\mathrm{ha}^{-1} \& \mathrm{FeSO}_{4} @ 10 \mathrm{~kg} \mathrm{ha}^{-1}+$ Foliar application of $0.5 \% \mathrm{ZnSO}_{4}$ and $\mathrm{FeSO}_{4}$ each recorded significantly higher zinc content (33.50 38.89 and $78.72 \mathrm{ppm}$ in grain, stover and total zinc content). The genotype $\mathrm{G}_{3}$ : $\mathrm{HFeZn}-113$ (high in $\mathrm{Zn} \& \mathrm{Fe}$ ) recorded significantly higher iron content $(177.18,166.71$ and $342.26 \mathrm{ppm}$ in grain and stover and total iron content respectively). Further among micronutrients application, significantly higher iron content was noticed in $\mathrm{M}_{7}$ : Soil application of $\mathrm{ZnSO}_{4} @ 15 \mathrm{~kg} \mathrm{ha}^{-1} \& \mathrm{FeSO}_{4} @ 10 \mathrm{~kg} \mathrm{ha}^{-1}+$ Foliar application of $0.5 \% \mathrm{ZnSO}_{4}$ and $\mathrm{FeSO}_{4}$ each recorded significantly higher iron content (195.45, 182.18 and $377.68 \mathrm{ppm}$ in grain, stover and total iron content). 


\section{Introduction}

Pearlmillet [Pennisetum glaucum (L.)] is the fifth most important cereal crop and widely grown in India during kharif. It is cultivated by economically poor farmers and provides staple food for the poor in short period in the relatively dry tracts of semi arid India. Now a days, in the context of changing climate, this crop is mostly identified as contingent crop in the country particularly in dry areas. Pearlmillet grain is the staple diet and nutritious source of vitamins, minerals, protein and carbohydrates, while its stover is a valuable livestock feed. In India, it is cultivated on an area of $7.30 \mathrm{~m}$ ha with the production of $8.73 \mathrm{~m} \mathrm{t}$, among which only 8.5 per cent cultivated area is under irrigation. Karnataka state stands $5^{\text {th }}$ position in area $(0.28 \mathrm{~m}$ ha $)$ and production $(0.29 \mathrm{~m} \mathrm{t})$ with the productivity of $1036 \mathrm{~kg} \mathrm{ha}^{-1}$ (Anon, 2014). The major area is confined to dry regions of northern Karnataka and generally grown as a rainfed crop and fits well in various cropping systems.

About half of the world's population suffers from micronutrient malnutrition a term used to refer any condition in which the body does not receive enough nutrients for proper function, including selenium $(\mathrm{Se})$, zinc $(\mathrm{Zn})$, iron $(\mathrm{Fe})$ and iodine (I), which is mainly associated with low dietary intake of micronutrients in diets with less diversity of food (Mayer et al., 2008). Zinc and iron deficiencies are welldocumented public health issue and an important soil constraint to crop production. Generally, there is a close geographical overlap between soil deficiency and human deficiency of $\mathrm{Zn}$ and $\mathrm{Fe}$, indicating a high requirement for increasing concentrations of these nutrients in food crops. Pearlmillet is a principle source of energy, protein, vitamins and minerals of millions of poorest people in region where it is cultivated. It general has 9 to13 per cent protein but large variation among genotype ranging from 6 to 21 per cent has been observed. Pearlmillet contains more calories than wheat, probably because of its higher oil content of 5 per cent of which 50 per cent are poly unsaturated fatty acid. It is rich in calcium, potassium, magnesium, iron, zinc, manganese, riboflavin, thiamine, niacin, lysine and tryptophan. Pearlmillet gluten is free and thus is the only grain that retains its alkaline properties after being cooked which is ideal for people with gluten allergies.

Agronomic biofortification providing $\mathrm{Zn}$ and Fe to plants by seed treatment and applying $\mathrm{Zn}$ or Fe fertilizers to soil and foliar appears to be important to ensure success of breeding efforts for increasing $\mathrm{Zn}$ and $\mathrm{Fe}$ concentration in grain. Fertilizer strategy could be a rapid solution to the problem and can be considered an important complementary approach to the on-going breeding programs. Fertilizer studies focusing specifically on increasing $\mathrm{Zn}$ and $\mathrm{Fe}$ concentration of grain are, however, very rare. The most effective method for increasing $\mathrm{Zn}$ and $\mathrm{Fe}$ in grain will be the combined application through soil and foliar method which results in an increase concentration of $\mathrm{Zn}$ and $\mathrm{Fe}$ in grain in addition to seed treatment. In most parts of the cereal growing areas, soils have, however, a variety of chemical and physical problems that significantly reduce availability of $\mathrm{Zn}$ and $\mathrm{Fe}$ to plant roots. Hence, the genetic capacity of the newly developed (biofortified) cultivars to absorb sufficient amount of $\mathrm{Zn}$ and $\mathrm{Fe}$ from soil and accumulate it in the grain may not be expressed to the full extent. It is, therefore, essential to have a short-term approach to improve $\mathrm{Zn}$ and $\mathrm{Fe}$ concentration in grains.

\section{Materials and Methods}

The field experiment was conducted at Agricultural College farm, Raichur, which is situated between $16^{\circ} 12^{\prime} \mathrm{N}$ latitude and $77^{\circ} 20^{\prime}$ E longitude with an altitude of 389 meters 
above the mean sea level and is located in zone II of Karnataka. The experiment was laid out in split plot design and comprised of two factors for study viz., genotypes (3 levels) and micronutrients application (7 levels). Main plot treatments: genotypes $(\mathrm{G})$ comprised viz., $\mathrm{G}_{1}$ : HFeZn-102 (low in $\mathrm{Zn} \& \mathrm{Fe}$ ), $\mathrm{G}_{2}$ : IP17720 (medium in $\mathrm{Zn} \& \mathrm{Fe}$ ) and $\mathrm{G}_{3}$ : HFeZn113 (high in $\mathrm{Zn} \& \mathrm{Fe}$ ). Subplot treatments: micronutrients application (M) comprised viz., $\mathrm{M}_{1}$ : Control, $\mathrm{M}_{2}$ : Seed treatment with $1 \%$ $\mathrm{ZnSO}_{4} \& \mathrm{FeSO}_{4}$ each, $\mathrm{M}_{3}$ : Soil application of $\mathrm{ZnSO}_{4} @ 15 \mathrm{~kg} \mathrm{ha}^{-1}$ and $\mathrm{FeSO}_{4} @ 10 \mathrm{~kg} \mathrm{ha}^{-1}$, $\mathrm{M}_{4}$ : Foliar application of $0.5 \% \mathrm{ZnSO}_{4}$ and $\mathrm{FeSO}_{4}$ each at 30 and 45 DAS, $\mathrm{M}_{5}$ : Seed treatment + Soil application $\left(M_{2}+M_{3}\right), M_{6}$ : Seed treatment + Foliar application $\left(\mathrm{M}_{2}+\mathrm{M}_{4}\right)$ and $\mathrm{M}_{7}$ : Soil application + Foliar application $\left(\mathrm{M}_{3}+\mathrm{M}_{4}\right)$. Treatments $\mathrm{M}_{1}$ to $\mathrm{M}_{7}$ includes, RDF: 50:25:00 kg N, $\mathrm{P}_{2} \mathrm{O}_{5}$ and $\mathrm{K}_{2} \mathrm{O} \mathrm{ha}^{-1}+$ FYM @ $2.5 \mathrm{t} \mathrm{ha}^{-1}$ ). The soils of the experimental site belong to medium deep black soil and clay texture, neutral in soil reaction (8.15) and low in electrical conductivity $\left(0.46 \mathrm{dSm}^{-1}\right)$. The organic carbon content was 0.69 per cent and low in available $\mathrm{N}\left(192.00 \mathrm{~kg} \mathrm{ha}^{-1}\right)$, medium in available phosphorus (22.90 $\left.\mathrm{kg} \mathrm{P}_{2} \mathrm{O}_{5} \mathrm{ha}^{-1}\right)$ and high in available potassium $\left(251.00 \mathrm{~kg} \mathrm{~K}_{2} \mathrm{O} \mathrm{ha}{ }^{-1}\right)$. DTPA extractable zinc $(0.55 \mathrm{ppm})$ and DTPA extractable iron $(3.72 \mathrm{ppm})$. The mean monthly meteorological data of rainfall, temperature and relative humidity during the period of experimentation (2016-17) recorded at the meteorological observatory of the MARS, Raichur.

\section{Results and Discussion}

In the present study, grain yield and stover yield of pearlmillet differed significantly due to agronomic biofortification the genotype $\mathrm{G}_{3}$ : HFeZn-113 (high in $\mathrm{Zn} \& \mathrm{Fe}$ ) recorded significantly higher grain and stover yield (1721 kg ha ${ }^{-1}$ and $4437 \mathrm{~kg} \mathrm{ha}^{-1}$, respectively) and it was on far with $\mathrm{G}_{2}$ : IP-17720 (medium in $\mathrm{Zn} \mathrm{\&} \mathrm{Fe)} \mathrm{(1719} \mathrm{kg} \mathrm{ha-1} \mathrm{and} 4255 \mathrm{~kg} \mathrm{ha}^{-1}$, respectively). Significantly higher grain and stover yield of pearlmillet was obtained in $\mathrm{M}_{7}$ : soil application of $\mathrm{ZnSO}_{4} @ 15 \mathrm{~kg} \mathrm{ha}^{-1}$ \& $\mathrm{FeSO}_{4} @ 10 \mathrm{~kg} \mathrm{ha}^{-1}+$ Foliar application of 0.5 $\% \mathrm{ZnSO}_{4}$ and $\mathrm{FeSO}_{4}\left(1904 \mathrm{~kg} \mathrm{ha}^{-1}\right.$ and 4611 $\mathrm{kg} \mathrm{ha}^{-1}$, respectively) which is on par with $\mathrm{M}_{5}$ : Seed treatment with $1 \% \mathrm{ZnSO}_{4} \& \mathrm{FeSO}_{4}+$ Soil application of $\mathrm{ZnSO}_{4} @ 15 \mathrm{~kg} \mathrm{ha}^{-1} \&$ $\mathrm{FeSO}_{4} @ 10 \mathrm{~kg} \mathrm{ha}^{-1}\left(1859 \mathrm{~kg} \mathrm{ha}^{-1}\right.$ and $4492 \mathrm{~kg}$ $\mathrm{ha}^{-1}$, respectively) followed by $\mathrm{M}_{3}$ : Soil application of $\mathrm{ZnSO}_{4} @ 15 \mathrm{~kg} \mathrm{ha}^{-1} \& \mathrm{FeSO}_{4}$ @ $10 \mathrm{~kg} \mathrm{ha}^{-1}$ (1770 kg ha ${ }^{-1}$ and $4351 \mathrm{~kg} \mathrm{ha}^{-1}$, respectively). Significantly lower pearlmillet grain and stover yield was recorded with control (1479 kg ha ${ }^{-1}$ and $3827 \mathrm{~kg} \mathrm{ha}^{-1}$, respectively) after $\mathrm{M}_{2}$ : Seed treatment with $1 \% \mathrm{ZnSO}_{4}$ and $\mathrm{FeSO}_{4}$ each (1582 kg ha ${ }^{-1}$ and $4132 \mathrm{~kg} \mathrm{ha}^{-1}$, respectively) and $\mathrm{M}_{4}$ : Foliar application of $0.5 \% \mathrm{ZnSO}_{4}$ and $\mathrm{FeSO}_{4}$ each (1657 kg ha ${ }^{-1}$ and $4163 \mathrm{~kg} \mathrm{ha}^{-1}$, respectively). Similar result was observed by Zeidan et al., (2010) and Esfahani et al., (2012).

The variation in the yield was due to the variation in the yield components viz., weight of ear head, length of ear head, number of ear heads and test weight. Higher grain yield of different pearlmillet genotypes is mainly due to higher weight of ear head in $\mathrm{G}_{3}$ : HFeZn-113 (high in $\mathrm{Zn} \mathrm{\&} \mathrm{Fe}$ ) (39.37 $\mathrm{g} \mathrm{plant}^{-1}$ ) when compared to other genoytpes. However, it was on par with $\mathrm{G}_{2}$ : IP-17720 (medium in $\mathrm{Zn}$ \& Fe) $\left(36.48 \mathrm{~g} \mathrm{plant}^{-1}\right)$. Whereas in case of micronutrients application higher weight of ear head of pearlmillet was recorded with $\mathrm{M}_{7}$ : Soil application of $\mathrm{ZnSO}_{4} @ 15 \mathrm{~kg} \mathrm{ha}^{-1}$ \& $\mathrm{FeSO}_{4} @ 10 \mathrm{~kg} \mathrm{ha}^{-1}+$ Foliar application of 0.5 $\% \mathrm{ZnSO}_{4}$ and $\mathrm{FeSO}_{4}$ each (48.09 $\mathrm{g} \mathrm{plant}^{-1}$ ) as compared to other treatments.

The genotype $\mathrm{G}_{3}$ : HFeZn-113 (high in $\mathrm{Zn}$ \& $\mathrm{Fe})$ recorded significantly higher length of ear head $(14.84 \mathrm{~cm})$ and it was on par with $\mathrm{G}_{2}$ : IP17720 (medium in $\mathrm{Zn} \mathrm{\&} \mathrm{Fe}$ ) $(14.09 \mathrm{~cm})$ and $\mathrm{G}_{1}$ : HFeZn-102 (low in Zn \& Fe) $(13.94 \mathrm{~cm}$ ). 
Table.1 Number of ear heads and test weight of pearlmillet genotypes as influenced by agronomic biofortification

\begin{tabular}{|c|c|c|c|c|c|c|c|c|}
\hline \multirow[t]{3}{*}{ Micronutrients application (M) } & \multicolumn{8}{|c|}{ Genotypes } \\
\hline & \multicolumn{4}{|c|}{ Number of ear heads plant ${ }^{-1}$} & \multicolumn{4}{|c|}{ Test weight (g) } \\
\hline & $\mathbf{G}_{1}$ & $\mathbf{G}_{2}$ & $\mathbf{G}_{3}$ & Mean & $\mathbf{G}_{1}$ & $\mathbf{G}_{2}$ & $\mathbf{G}_{3}$ & Mean \\
\hline$M_{1}$ : Control & 1.01 & 1.11 & 1.50 & 1.22 & 10.15 & 12.52 & 13.08 & 11.92 \\
\hline $\mathrm{M}_{2}$ : Seed treatment with $1 \% \mathrm{ZnSO}_{4} \& \mathrm{FeSO}_{4}$ each & 2.08 & 2.01 & 3.05 & 2.31 & 13.72 & 14.19 & 14.03 & 13.98 \\
\hline $\begin{array}{l}\mathrm{M}_{3} \text { : Soil application of } \mathrm{ZnSO}_{4} @ 15 \mathrm{~kg} \mathrm{ha}^{-1} \& \mathrm{FeSO}_{4} @ 10 \\
\mathrm{~kg} \mathrm{ha}^{-1}\end{array}$ & 2.02 & 3.02 & 2.01 & 2.30 & 15.63 & 15.12 & 17.73 & 16.16 \\
\hline $\begin{array}{l}\mathrm{M}_{4} \text { : Foliar application of } 0.5 \% \mathrm{ZnSO}_{4} \& \mathrm{FeSO}_{4} \text { each at } 30 \\
\text { and } 45 \text { DAS }\end{array}$ & 3.07 & 2.03 & 4.02 & 3.15 & 14.90 & 10.51 & 13.73 & 13.05 \\
\hline$M_{5}:$ Seed treatment + Soil application & 4.12 & 4.01 & 3.01 & 3.74 & 17.83 & 17.98 & 18.04 & 17.95 \\
\hline$M_{6}:$ Seed treatment + Foliar application & 3.09 & 2.03 & 4.02 & 3.20 & 14.88 & 13.97 & 15.84 & 14.89 \\
\hline$M_{7}$ : Soil application + Foliar application & 4.01 & 6.01 & 5.03 & 5.45 & 18.33 & 18.58 & 18.63 & 18.51 \\
\hline Mean & 2.73 & 2.96 & 3.21 & - & 15.06 & 14.70 & 15.87 & - \\
\hline For comparing means of & \multicolumn{2}{|c|}{ S.Em \pm} & \multicolumn{2}{|c|}{ C.D. at $5 \%$} & \multicolumn{2}{|c|}{ S.Em \pm} & \multicolumn{2}{|c|}{ C.D. at $5 \%$} \\
\hline Genotypes (G) & \multicolumn{2}{|c|}{0.82} & \multicolumn{2}{|c|}{2.50} & \multicolumn{2}{|c|}{0.32} & \multicolumn{2}{|c|}{1.00} \\
\hline Micronutrients application (M) & \multicolumn{2}{|c|}{0.22} & \multicolumn{2}{|c|}{0.70} & \multicolumn{2}{|c|}{0.13} & \multicolumn{2}{|c|}{0.45} \\
\hline$M$ at the same level of $G$ & \multicolumn{2}{|c|}{1.33} & \multicolumn{2}{|c|}{ NS } & \multicolumn{2}{|c|}{1.16} & \multicolumn{2}{|c|}{ NS } \\
\hline$G$ at the same or different levels of $M$ & \multicolumn{2}{|c|}{0.95} & \multicolumn{2}{|c|}{ NS } & \multicolumn{2}{|c|}{1.02} & \multicolumn{2}{|c|}{ NS } \\
\hline
\end{tabular}

Note: 1. G $\mathbf{G}_{\mathbf{1}}$ : HFeZn-102 (low in Zn \& Fe), $\mathbf{G}_{2}$ : IP-17720 (medium in Zn \& Fe) and $\mathbf{G}_{3}$ : HFeZn-113 (high in Zn \& Fe). NS - Non Significant Note: $2 . R D F$ is common to all the treatment from $M_{1}$ and $M_{7}$ 
Table.2 Length of ear head, weight of ear head, grain weight of pearlmillet genotypes as influenced by agronomic biofortification

\begin{tabular}{|c|c|c|c|c|c|c|c|c|c|c|c|c|}
\hline \multirow[t]{3}{*}{ Micronutrients application (M) } & \multicolumn{12}{|c|}{ Genotypes (G) } \\
\hline & \multicolumn{4}{|c|}{ Length of ear head $(\mathrm{cm})$} & \multicolumn{4}{|c|}{ Weight of ear head (g plant ${ }^{-1}$ ) } & \multicolumn{4}{|c|}{ Grain weight (g plant ${ }^{-1}$ ) } \\
\hline & $\mathbf{G}_{\mathbf{1}}$ & $\mathbf{G}_{2}$ & $\mathbf{G}_{\mathbf{3}}$ & Mean & $\mathbf{G}_{\mathbf{1}}$ & $\mathbf{G}_{\mathbf{2}}$ & $\mathbf{G}_{\mathbf{3}}$ & Mean & $\mathbf{G}_{\mathbf{1}}$ & $\mathbf{G}_{\mathbf{2}}$ & $\mathbf{G}_{\mathbf{3}}$ & Mean \\
\hline $\mathrm{M}_{1}$ : Control & 7.47 & 8.38 & 8.51 & 8.12 & 21.00 & 26.83 & 28.92 & 25.59 & 21.76 & 19.40 & 20.72 & 20.63 \\
\hline $\begin{array}{l}\mathrm{M}_{2}: \text { Seed treatment with } 1 \% \mathrm{ZnSO}_{4} \& \mathrm{FeSO}_{4} \\
\text { each }\end{array}$ & 12.39 & 13.45 & 13.42 & 13.09 & 34.25 & 33.17 & 31.83 & 33.08 & 23.65 & 26.30 & 27.70 & 25.88 \\
\hline $\begin{array}{l}\mathrm{M}_{3} \text { : Soil application of } \mathrm{ZnSO}_{4} @ 15 \mathrm{~kg} \mathrm{ha}^{-1} \& \\
\mathrm{FeSO}_{4} @ 10 \mathrm{~kg} \mathrm{ha}^{-1}\end{array}$ & 15.95 & 15.88 & 11.69 & 14.51 & 44.55 & 31.58 & 46.83 & 40.99 & 35.80 & 30.33 & 38.75 & 34.96 \\
\hline $\begin{array}{l}\mathrm{M}_{4}: \begin{array}{l}\text { Foliar application of } 0.5 \% \\
\mathrm{FeSO}_{4}\end{array} \\
\text { each at } 30 \text { and } 45 \mathrm{DAS}\end{array}$ & 11.89 & 14.00 & 14.43 & 13.44 & 30.50 & 34.17 & 34.83 & 33.17 & 29.60 & 30.10 & 30.68 & 30.13 \\
\hline$M_{5}:$ Seed treatment + Soil application & 18.21 & 18.75 & 19.76 & 18.91 & 45.83 & 47.03 & 48.10 & 46.99 & 36.80 & 38.13 & 40.13 & 38.36 \\
\hline$M_{6}:$ Seed treatment + Foliar application & 12.47 & 8.57 & 13.60 & 11.55 & 33.67 & 34.37 & 35.97 & 34.67 & 32.95 & 33.17 & 37.03 & 34.38 \\
\hline $\mathbf{M}_{7}$ : Soil application + Foliar application & 19.17 & 19.62 & 22.44 & 20.41 & 46.96 & 48.20 & 49.10 & 48.09 & 37.95 & 39.88 & 41.83 & 39.89 \\
\hline Mean & 13.94 & 14.09 & 14.84 & - & 36.68 & 36.48 & 39.37 & - & 31.22 & 31.05 & 33.84 & - \\
\hline For comparing means of & \multicolumn{2}{|c|}{ S.Em \pm} & \multicolumn{2}{|c|}{ C.D. at $5 \%$} & \multicolumn{2}{|c|}{ S.Em \pm} & \multicolumn{2}{|c|}{ C.D. at $5 \%$} & \multicolumn{2}{|c|}{ S.Em \pm} & \multicolumn{2}{|c|}{ C.D. at $5 \%$} \\
\hline Genotypes (G) & \multicolumn{2}{|c|}{1.24} & \multicolumn{2}{|c|}{4.88} & \multicolumn{2}{|c|}{1.67} & \multicolumn{2}{|c|}{6.55} & \multicolumn{2}{|c|}{1.12} & \multicolumn{2}{|c|}{4.41} \\
\hline Micronutrients application (M) & \multicolumn{2}{|c|}{0.82} & \multicolumn{2}{|c|}{2.35} & \multicolumn{2}{|c|}{1.07} & \multicolumn{2}{|c|}{3.06} & \multicolumn{2}{|c|}{0.21} & \multicolumn{2}{|c|}{0.60} \\
\hline$M$ at the same level of $G$ & \multicolumn{2}{|c|}{1.42} & \multicolumn{2}{|c|}{ NS } & \multicolumn{2}{|c|}{1.85} & \multicolumn{2}{|c|}{ NS } & \multicolumn{2}{|c|}{0.36} & \multicolumn{2}{|c|}{ NS } \\
\hline$G$ at the same or different levels of $M$ & \multicolumn{2}{|c|}{2.00} & \multicolumn{2}{|c|}{ NS } & \multicolumn{2}{|c|}{2.61} & \multicolumn{2}{|c|}{ NS } & \multicolumn{2}{|c|}{0.51} & \multicolumn{2}{|c|}{ NS } \\
\hline
\end{tabular}

Note: 1. G $\mathbf{G}_{1}$ : HFeZn-102 (low in Zn \& Fe), $\mathbf{G}_{2}$ : IP-17720 (medium in Zn \& Fe) and $\mathbf{G}_{3}$ : HFeZn-113 (high in Zn \& Fe). NS - Non Significant Note: 2 . $R D F$ is common to all the treatment from $M_{1}$ and $M_{7}$ 
Table.3 Grain yield, stover yield and harvest index of pearlmillet genotypes as influenced by genotypes and agronomic biofortification

\begin{tabular}{|c|c|c|c|c|c|c|c|c|c|c|c|c|}
\hline \multirow[t]{3}{*}{ Micronutrients application (M) } & \multicolumn{12}{|c|}{ Genotypes (G) } \\
\hline & \multicolumn{4}{|c|}{ Grain yield $\left(\mathrm{kg} \mathrm{ha}^{-1}\right)$} & \multicolumn{4}{|c|}{ Stover yield (kg ha $\left.{ }^{-1}\right)$} & \multicolumn{4}{|c|}{ Harvest index (\%) } \\
\hline & $\mathbf{G}_{1}$ & $\mathbf{G}_{2}$ & $\mathbf{G}_{3}$ & Mean & $\mathbf{G}_{1}$ & $\mathbf{G}_{\mathbf{2}}$ & $\mathbf{G}_{3}$ & Mean & $\mathbf{G}_{1}$ & $\mathbf{G}_{\mathbf{2}}$ & $\mathbf{G}_{3}$ & Mean \\
\hline $\mathrm{M}_{1}$ : Control & 1477 & 1479 & 1483 & 1479 & 3707 & 3831 & 3943 & 3827 & 28.49 & 27.85 & 27.33 & 27.87 \\
\hline $\begin{array}{l}\mathrm{M}_{2}: \text { Seed treatment with } 1 \% \mathrm{ZnSO}_{4} \& \mathrm{FeSO}_{4} \\
\text { each }\end{array}$ & 1581 & 1581 & 1585 & 1582 & 3999 & 4112 & 4286 & 4132 & 28.33 & 27.77 & 27.00 & 27.69 \\
\hline $\begin{array}{l}\mathrm{M}_{3}: \text { Soil application of } \mathrm{ZnSO}_{4} @ 15 \mathrm{~kg} \mathrm{ha}^{-1} \& \\
\mathrm{FeSO}_{4} @ 10 \mathrm{~kg} \mathrm{ha}^{-1}\end{array}$ & 1764 & 1772 & 1775 & 1770 & 4146 & 4374 & 4532 & 4351 & 29.85 & 28.83 & 28.14 & 28.92 \\
\hline $\begin{array}{l}\mathrm{M}_{4}: \text { Foliar application of } 0.5 \% \mathrm{ZnSO}_{4} \& \mathrm{FeSO}_{4} \\
\text { each at } 30 \text { and } 45 \mathrm{DAS}\end{array}$ & 1644 & 1650 & 1678 & 1657 & 4001 & 4125 & 4362 & 4163 & 29.12 & 28.57 & 27.78 & 28.47 \\
\hline$M_{5}$ : Seed treatment + Soil application & 1855 & 1870 & 1852 & 1859 & 4167 & 4494 & 4815 & 4492 & 30.80 & 29.38 & 27.78 & 29.27 \\
\hline$M_{6}:$ Seed treatment + Foliar application & 1741 & 1738 & 1765 & 1748 & 4167 & 4294 & 4224 & 4228 & 29.47 & 28.81 & 29.47 & 29.25 \\
\hline $\mathbf{M}_{7}$ : Soil application + Foliar application & 1859 & 1940 & 1912 & 1904 & 4377 & 4557 & 4898 & 4611 & 29.81 & 29.86 & 28.08 & 29.22 \\
\hline Mean & 1703 & 1719 & 1721 & - & 4081 & 4255 & 4437 & - & 29.44 & 28.77 & 27.95 & - \\
\hline For comparing means of & \multicolumn{2}{|c|}{ S.Em \pm} & \multicolumn{2}{|c|}{ C.D. at $5 \%$} & \multicolumn{2}{|c|}{ S.Emt } & \multicolumn{2}{|c|}{ C.D. at $5 \%$} & \multicolumn{2}{|c|}{ S.Em \pm} & \multicolumn{2}{|c|}{ C.D. at $5 \%$} \\
\hline Genotypes (G) & \multicolumn{2}{|c|}{25.92} & \multicolumn{2}{|c|}{101.78} & \multicolumn{2}{|c|}{38.78} & \multicolumn{2}{|c|}{152.26} & \multicolumn{2}{|c|}{0.00} & \multicolumn{2}{|c|}{0.01} \\
\hline Micronutrients application (M) & \multicolumn{2}{|c|}{21.53} & \multicolumn{2}{|c|}{61.75} & \multicolumn{2}{|c|}{39.74} & \multicolumn{2}{|c|}{113.97} & \multicolumn{2}{|c|}{0.00} & \multicolumn{2}{|c|}{0.01} \\
\hline$M$ at the same level of $G$ & \multicolumn{2}{|c|}{37.29} & \multicolumn{2}{|c|}{ NS } & \multicolumn{2}{|c|}{68.83} & \multicolumn{2}{|c|}{ NS } & \multicolumn{2}{|c|}{0.00} & \multicolumn{2}{|c|}{ NS } \\
\hline G at the same or different levels of $M$ & \multicolumn{2}{|c|}{52.74} & \multicolumn{2}{|c|}{ NS } & \multicolumn{2}{|c|}{97.34} & \multicolumn{2}{|c|}{ NS } & \multicolumn{2}{|c|}{0.01} & \multicolumn{2}{|c|}{ NS } \\
\hline
\end{tabular}

Note: 1. $\mathbf{G}_{1}: \mathrm{HFeZn}-102$ (low in Zn \& Fe), $\mathbf{G}_{2}$ : IP-17720 (medium in Zn \& Fe) and $\mathbf{G}_{3}:$ HFeZn-113 (high in Zn \& Fe). NS - Non Significant Note: 2 . RDF is common to all the treatment from $\mathrm{M}_{1}$ and $\mathrm{M}_{7}$ 
Table.4 Zinc content in pearlmillet grain, stover and total zinc content as influenced by genotypes and agronomic biofortification

\begin{tabular}{|c|c|c|c|c|c|c|c|c|c|c|c|c|}
\hline \multirow[t]{2}{*}{ Micronutrients application (M) } & \multicolumn{12}{|c|}{ Genotypes (G) } \\
\hline & $\mathbf{G}_{1}$ & $\mathbf{G}_{2}$ & $\mathbf{G}_{3}$ & Mean & $\mathbf{G}_{1}$ & $\mathbf{G}_{\mathbf{2}}$ & $\mathbf{G}_{3}$ & Mean & $\mathbf{G}_{1}$ & $\mathbf{G}_{2}$ & $\mathbf{G}_{\mathbf{3}}$ & Mean \\
\hline $\begin{array}{l}\mathrm{M}_{2}: \text { Seed treatment with } 1 \% \mathrm{ZnSO}_{4} \& \mathrm{FeSO}_{4} \\
\text { each }\end{array}$ & 22.27 & 25.27 & 27.77 & 25.10 & 31.58 & 34.58 & 38.83 & 35.00 & 53.85 & 59.85 & 66.60 & 60.10 \\
\hline $\begin{array}{l}\mathrm{M}_{4}: \text { Foliar application of } 0.5 \% \mathrm{ZnSO}_{4} \& \mathrm{FeSO}_{4} \\
\text { each at } 30 \text { and } 45 \text { DAS }\end{array}$ & 23.33 & 22.43 & 22.67 & 22.81 & 31.75 & 35.58 & 37.75 & 35.03 & 55.08 & 58.02 & 60.42 & $\mathbf{5 7 . 8 4}$ \\
\hline$M_{5}:$ Seed treatment + Soil application & 29.00 & 32.67 & 35.67 & 32.44 & 40.33 & 40.75 & 43.33 & 42.14 & 69.75 & 73.00 & 78.00 & 73.58 \\
\hline$M_{6}:$ Seed treatment + Foliar application & 24.77 & 27.50 & 30.17 & 27.48 & 32.67 & 35.67 & 38.58 & 35.64 & 57.43 & 63.17 & 68.75 & 63.12 \\
\hline Genotypes (G) & \multicolumn{2}{|c|}{0.31} & \multicolumn{2}{|c|}{1.23} & \multicolumn{2}{|c|}{0.97} & \multicolumn{2}{|c|}{3.79} & \multicolumn{2}{|c|}{1.01} & \multicolumn{2}{|c|}{6.95} \\
\hline Micronutrients application (M) & \multicolumn{2}{|c|}{0.66} & \multicolumn{2}{|c|}{1.90} & \multicolumn{2}{|c|}{0.66} & \multicolumn{2}{|c|}{1.90} & \multicolumn{2}{|c|}{0.95} & \multicolumn{2}{|c|}{2.71} \\
\hline$M$ at the same level of $G$ & \multicolumn{2}{|c|}{1.15} & \multicolumn{2}{|c|}{ NS } & \multicolumn{2}{|c|}{1.15} & \multicolumn{2}{|c|}{ NS } & \multicolumn{2}{|c|}{1.64} & \multicolumn{2}{|c|}{ NS } \\
\hline$G$ at the same or different levels of $M$ & \multicolumn{2}{|c|}{1.62} & \multicolumn{2}{|c|}{ NS } & \multicolumn{2}{|c|}{1.63} & \multicolumn{2}{|c|}{ NS } & \multicolumn{2}{|c|}{2.32} & \multicolumn{2}{|c|}{ NS } \\
\hline
\end{tabular}

Note: 1. $\mathbf{G}_{\mathbf{1}}: \mathrm{HFeZn}-102$ (low in Zn \& Fe), $\mathbf{G}_{\mathbf{2}}$ : IP-17720 (medium in Zn \& Fe) and $\mathbf{G}_{3}$ : HFeZn-113 (high in Zn \& Fe). NS - Non Significant Note: 2. $R D F$ is common to all the treatment from $M_{1}$ and $M_{7}$ 
Table. 5 Iron content in pearlmillet grain, stover and total iron content as influenced by genotypes and agronomic biofortification

\begin{tabular}{|c|c|c|c|c|c|c|c|c|c|c|c|c|}
\hline \multirow[t]{3}{*}{ Micronutrients application (M) } & \multicolumn{12}{|c|}{ Genotypes (G) } \\
\hline & \multicolumn{4}{|c|}{ Grain (ppm) } & \multicolumn{4}{|c|}{ Stover (ppm) } & \multicolumn{4}{|c|}{ Total Fe concentration (ppm) } \\
\hline & $\mathbf{G}_{1}$ & $\mathbf{G}_{2}$ & $\mathbf{G}_{3}$ & Mean & $\mathbf{G}_{1}$ & $\mathbf{G}_{2}$ & $\mathbf{G}_{3}$ & Mean & $\mathbf{G}_{1}$ & $\mathbf{G}_{2}$ & $\mathbf{G}_{3}$ & Mean \\
\hline $\mathrm{M}_{1}:$ Control & 142.18 & 144.24 & 146.80 & 144.41 & 124.31 & 128.31 & 130.31 & 127.65 & 266.54 & 272.41 & 277.10 & 272.12 \\
\hline $\mathrm{M}_{2}:$ Seed treatment with $1 \% \mathrm{ZnSO}_{4} \& \mathrm{FeSO}_{4}$ Each & 165.21 & 168.14 & 168.15 & 167.22 & 152.23 & 158.24 & 155.24 & 155.25 & 317.45 & 326.35 & 323.38 & 322.35 \\
\hline $\begin{array}{l}\mathrm{M}_{3} \text { : Soil application of } \mathrm{ZnSO}_{4} @ 15 \mathrm{~kg} \mathrm{ha}^{-1} \& \mathrm{FeSO}_{4} \\
\text { @10 kg ha }\end{array}$ & 185.24 & 185.12 & 185.18 & 185.20 & 171.24 & 172.29 & 175.12 & 172.90 & 356.36 & 357.41 & 360.31 & 358.15 \\
\hline $\begin{array}{l}\mathrm{M}_{4}: \text { Foliar application of } 0.5 \% \mathrm{ZnSO}_{4} \& \mathrm{FeSO}_{4} \text { each } \\
\text { at } 30 \text { and } 45 \mathrm{DAS}\end{array}$ & 172.15 & 172.15 & 175.20 & 173.24 & 162.21 & 161.15 & 165.18 & 162.88 & 334.38 & 333.39 & 340.25 & 336.09 \\
\hline$M_{5}:$ Seed treatment + Soil application & 189.16 & 191.13 & 188.10 & 190.12 & 174.18 & 175.14 & 179.19 & 176.75 & 363.45 & 366.32 & 366.14 & 366.65 \\
\hline$M_{6}:$ Seed treatment + Foliar application & 176.75 & 178.25 & 179.18 & 178.14 & 165.14 & 168.32 & 171.17 & 168.21 & 342.21 & 346.25 & 350.21 & 346.25 \\
\hline$M_{7}:$ Soil application + Foliar application & 194.12 & 195.32 & 197.21 & 195.45 & 180.22 & 182.18 & 184.23 & 182.18 & 374.45 & 377.12 & 381.14 & 377.68 \\
\hline Mean & 175.10 & 176.31 & 177.18 & - & 161.43 & 163.72 & 166.71 & - & 336.34 & 340.10 & 342.26 & - \\
\hline For comparing means of & \multicolumn{2}{|c|}{ S.Em \pm} & \multicolumn{2}{|c|}{ C.D. at $5 \%$} & \multicolumn{2}{|c|}{ S.Em \pm} & \multicolumn{2}{|c|}{ C.D. at $5 \%$} & \multicolumn{2}{|c|}{ S.Em \pm} & \multicolumn{2}{|c|}{ C.D. at $5 \%$} \\
\hline Genotypes (G) & \multicolumn{2}{|c|}{1.46} & \multicolumn{2}{|c|}{5.73} & \multicolumn{2}{|c|}{1.41} & \multicolumn{2}{|c|}{5.54} & \multicolumn{2}{|c|}{2.83} & \multicolumn{2}{|c|}{11.10} \\
\hline Micronutrients application (M) & \multicolumn{2}{|c|}{0.24} & \multicolumn{2}{|c|}{0.68} & \multicolumn{2}{|c|}{0.58} & \multicolumn{2}{|c|}{1.66} & \multicolumn{2}{|c|}{0.59} & \multicolumn{2}{|c|}{1.69} \\
\hline M at the same level of $G$ & \multicolumn{2}{|c|}{0.41} & \multicolumn{2}{|c|}{ NS } & \multicolumn{2}{|c|}{1.00} & \multicolumn{2}{|c|}{ NS } & \multicolumn{2}{|c|}{1.02} & \multicolumn{2}{|c|}{ NS } \\
\hline$G$ at the same or different levels of $M$ & \multicolumn{2}{|c|}{0.58} & \multicolumn{2}{|c|}{ NS } & \multicolumn{2}{|c|}{1.42} & \multicolumn{2}{|c|}{ NS } & \multicolumn{2}{|c|}{1.45} & \multicolumn{2}{|c|}{ NS } \\
\hline
\end{tabular}

Note: 1. $\mathbf{G}_{1}: \mathrm{HFeZn}-102$ (low in Zn \& Fe), $\mathbf{G}_{2}$ : IP-17720 (medium in Zn \& Fe) and $\mathbf{G}_{\mathbf{3}}$ : HFeZn-113 (high in Zn \& Fe). NS - Non Significant Note: $2 . R D F$ is common to all the treatment from $M_{1}$ and $M_{7}$ 
Similarly micronutrients application higher length of ear head of pearlmillet was recorded with $\mathrm{M}_{7}$ : Soil application + Foliar application $(20.41 \mathrm{~cm})$ as compared to control $(8.12 \mathrm{~cm})$. Similarly higher grain yield of different pearlmillet genotypes is mainly due to number of ear heads. The genotype $\mathrm{G}_{3}$ : HFeZn-113 (high in $\mathrm{Zn} \& \mathrm{Fe}$ ) recorded significantly higher number of ear heads (3.21 plant $\left.^{-1}\right)$ and on far with $\mathrm{G}_{2}$ : IP-17720 (medium in $\mathrm{Zn} \&$ $\mathrm{Fe}$ ) (2.96 plant $^{-1}$ ) and $\mathrm{G}_{1}$ : HFeZn-102 (low in $\mathrm{Zn} \& \mathrm{Fe})\left(2.73\right.$ plant $\left.^{-1}\right)$. Among the micronutrient application higher number of ear heads was recorded with $\mathrm{M}_{7}$ : Soil application of $\mathrm{ZnSO}_{4} @ 15 \mathrm{~kg} \mathrm{ha}^{-1} \& \mathrm{FeSO}_{4}$ @ $10 \mathrm{~kg} \mathrm{ha}^{-1}+$ Foliar application of $0.5 \%$ $\mathrm{ZnSO}_{4}$ and $\mathrm{FeSO}_{4}\left(5.03\right.$ plant $\left.^{-1}\right)$ as compared to the other treatments. The increase in the yield attributes could be due to continuous supply of micronutrients $(\mathrm{Zn}$ and $\mathrm{Fe}$ ) to the crop. $\mathrm{Zn}$ and $\mathrm{Fe}$ are part of the photosynthesis, assimilation and translocation of photosynthates from source (leaves) to sink (ear head) (Singh et al., 1995), Similar trend was noticed by Adsul et al., (2011) and Olusengun et al., (2014).

The content of zinc in pearlmillet grain and stover differed significantly. The genotype $\mathrm{G}_{3}$ : HFeZn-113 (high in $\mathrm{Zn} \& \mathrm{Fe}$ ) recorded significantly higher zinc content $(29.56,38.89$ and $66.45 \mathrm{ppm}$ in grain, stover and total zinc content respectively), as compared to other genotypes. Among micronutrients application, significantly higher zinc content was observed in $\mathrm{M}_{7}$ : Soil application of $\mathrm{ZnSO}_{4}$ @ $15 \mathrm{~kg} \mathrm{ha}^{-1}$ \& $\mathrm{FeSO}_{4} @ 10 \mathrm{~kg} \mathrm{ha}^{-1}+$ Foliar application of $0.5 \% \mathrm{ZnSO}_{4}$ and $\mathrm{FeSO}_{4}$ each recorded significantly higher zinc content (33.50 38.89 and $78.72 \mathrm{ppm}$ in grain, stover and total zinc content) as compared to other treatments except $\mathrm{M}_{5}$ : Seed treatment + Soil application (32.44, 42.14 and $73.58 \mathrm{ppm}$ in grain, stover and total zinc content, respectively), whereas in case of iron content in pearlmillet genotypes in grain, stover and total iron content differed significantly. The genotype $\mathrm{G}_{3}$ : HFeZn-113 (high in $\mathrm{Zn} \& \mathrm{Fe}$ ) recorded significantly higher iron content (177.18, 166.71 and $342.26 \mathrm{ppm}$ in grain and stover and total iron content respectively). Further among micronutrients application, significantly higher iron content was noticed in $\mathrm{M}_{7}$ : Soil application of $\mathrm{ZnSO}_{4} @ 15 \mathrm{~kg} \mathrm{ha}^{-1}$ \& $\mathrm{FeSO}_{4} @ 10 \mathrm{~kg} \mathrm{ha}^{-1}+$ Foliar application of $0.5 \% \quad \mathrm{ZnSO}_{4}$ and $\mathrm{FeSO}_{4}$ each recorded significantly higher iron content (195.45, 182.18 and $377.68 \mathrm{ppm}$ in grain, stover and total iron content), as compared to other treatments except $\mathrm{M}_{5}$ : Seed treatment + Soil application (190.12, 176.75 and $366.65 \mathrm{ppm}$ in grain, stover and total iron content, respectively). Similar result was observed by yang et al., (2011). This may due to increase in grain yield due to increase the content of these micronutrient ( $\mathrm{Zn}$ and $\mathrm{Fe}$ ), the effect of soil and foliar application of $\mathrm{ZnSO}_{4}$ and $\mathrm{FeSO}_{4}$ for better absorption and enhancing the availability these micronutrients.

\section{References}

Adsul, P. B., Anuradha, P., Ganesh, G., Ajeet, P. and Shiekh, S. S., 2011, Uptake of $\mathrm{N}, \mathrm{P}, \mathrm{K}$ and yield of kharif sorghum as influenced by soil and foliar application of micronutrients. Bioinfolet., 11(2): 578-582.

Anonymous, 2014, Agricultural statistics at a glance, Government of India, Ministry of Agriculture, Department of Agriculture and Cooperation, Directorate of Economics and Statistics. p.63.

Cakmak, I., Velu, G., Monasterio, O. I., Hao, Y. and Singh, R. P., 2010b, Biofortification strategies to increase grain zinc and iron concentrations in wheat. J. Cereal Sci., 59:365-372.

Esfahani, A., Hemmatollah, P. and Yousuf, N., 2012, Effect of iron, zinc and silicon application on quantitative 
parameters of rice. Int. J. Alli. Sci., 3 (5):529-533.

Habib, M., 2009, Effect of foliar application of $\mathrm{Zn}$ and $\mathrm{Fe}$ on wheat yield and quality. African J. Biotech., 8 (24): 6795-6798.

Mahmed, M. F., Thalooth, A. T. and Khalifa, R. M., 2010, Effect of foliar spraying with uniconazole and micronutrients on yield and nutrient uptake of wheat plants grown under saline condition. American J. Sci., 6 (8): 398-404.

Mayer, J. E., Pfeiffer, W. H. and Beyer, P., 2008, Biofortified crops to alleviate micronutrient malnutrition. Curr. Opin. Plant Biol., 11: 166-170.

Olusengun, A. and Meki, C., 2014, Soil application of zinc to maize and wheat grown on a zambian Alfisol. African J. Agril. Res., 9(11): 963-970.

Sandhya Rani, Y. and Patro T. S. K., 2014, Evaluation of effect of zinc biofortification on crop growth and grain yield in finger millet (Eleusine coracana). Int. J. Food, Agric. Veter. Sci., 4(2):146-148.
Singh, D. and Singh, R. N., 1995, Effect of potassium, zinc and sulphur on growth characters, yield attributes and yield of soybean (Glycine max L.). Indian J. Agron., 40(2): 223-227.

Yang, X. W., Tian, X. H., Gale, W. J., Cao, Y. X., Lu, X. C. and Zhao, A. Q., 2011, Effect of soil and foliar zinc application on zinc concentration and bioavailability in wheat grain grown on potentially zinc deficient soils. Cereal Res. Commun., 39:535-543.

Yilmaz, A, Ekiz, H., Gultekin, I., Torun, B., Barut, H., Karanlik, S. and Cakmak, I., 1998, Effect of seed zinc content on grain yield and zinc concentration of wheat grown in zinc deficient calcareous soils. J. Plant Nutr., 21 : 2257-2264.

Zeidan, M. S., Mohamad, M. S. and Hamouda, H. A., 2010, Effect of foliar fertilization of $\mathrm{Fe}, \mathrm{Mn}$ and $\mathrm{Zn}$ on wheat yield and quality in low sandy soil fertility. World J. Agric. Sci., 6 (6): 696-699.

\section{How to cite this article:}

Sharanappa, H. S. Latha, B. K. Desai, B. G.Koppalkar and Ravi M. V. 2019. Effect of Agronomic Biofortification with Zinc and Iron on Yield and Quality of Pearlmillet [Pennisetum glaucum (L.)] Genotypes. Int.J.Curr.Microbiol.App.Sci. 8(09): 1312-1321. doi: https://doi.org/10.20546/ijcmas.2019.809.150 